\title{
Enhanced stability and fluidity in droplet on hydrogel bilayers for measuring membrane protein diffusion - Supporting Information
}

\author{
James R. Thompson ${ }^{\dagger}$, Andrew J. Heron ${ }^{\dagger}$, Yusdi Santoso ${ }^{\S}$, Mark I. Wallace ${ }^{\dagger *}$
}

\section{Methods}

\section{$1.1 \quad$ Materials}

Unless otherwise stated, all chemicals were from Sigma (Sigma-Aldrich Ltd, Gillingham, U.K.). Triton X-100 detergent, acrylamide and $\beta$-mercaptoethanol (2-ME) were from Bio-Rad (Bio-Rad Laboratories, Hercules, CA). Fluorescently-labeled lipid, N-(6-tetramethylrhodaminethiocarbamoyl)-1,2-dihexadecanoylsn-glycero-3-phosphoethanolamine (TRITC-DHPE) was from Invitrogen (Invitrogen Corporation, Carlsbad, CA). E. coli bacteria stocks were from Promega (Promega, Madison, WI). Doubly-deionised 18 $\mathrm{M} \Omega \mathrm{cm}^{-1}$ water was used throughout (Millipore Corporation, Billerica, MA). Dioxane-free isopropyl$\beta$-D-thiogalactopyranoside (IPTG) was from Calbiochem (EMD Biosciences Inc, San Diego, CA). All chromatography equipment and Cy-dyes were from GE Healthcare (GE Healthcare Life Sciences, Amersham, U.K.). $0.2 \mu \mathrm{m}$ cellulose acetate filters were from Rainin (Rainin Instrument LLC, Oakland, CA).

\subsection{Supported Lipid Bilayers}

SLBs were formed by vesicle deposition on glass coverslips as described previously. ${ }^{1}$ Briefly, coverslips were cleaned by exposure to $\mathrm{O}_{2}$ plasma, 24 hour immersion in $2 \mathrm{M} \mathrm{KOH}$ followed by extensive washing with water and drying with $\mathrm{N}_{2}$ gas. Phospholipid vesicles containing $10 \mathrm{pM}$ TRITC-DHPE and $1.27 \mathrm{mM}$ DOPC lipids were prepared by dilution from stock in dichloromethane, followed by mixing to give the desired ratio. Solvent was evaporated with $\mathrm{N}_{2}$ gas and the lipids were resuspended in TE buffer (10 mM Tris-HCl, $1 \mathrm{mM}$ 
EDTA, pH 8.0). The suspension was then sonicated for 2 hours to transparency. $20 \mu \mathrm{L}$ of this solution was pipetted onto a cleaned coverslip. After 5 minutes the coverslip was washed repeatedly with TE buffer prior to microscopy.

\subsection{Droplet on Hydrogel Bilayers}

Lipids for fluorophore-containing DHBs were prepared as above by dilution and mixing from stock in dichloromethane. After solvent evaporation, lipids were redissolved in hexadecane, yielding a solution of approximately 10 pM TRITC-DHPE and $6.35 \mathrm{mM}$ DOPC lipids. $10 \mu \mathrm{L}$ of molten $1.5 \%$ agarose in TE buffer was deposited on a coverslip and dragged across the surface using another cleaned coverslip to create a thin film. This was air-dried, leaving an ultra-thin coating of dehydrated agarose upon the coverslip surface. A micro-channel device made of polymethyl methacrylate (PMMA) was placed on top of the dried agarose layer. The machined PMMA device consists of a series of wells surrounded by deeper channels which can contact the underlying substrate agarose. These channels serve as a conduit for passing rehydrating molten agarose around the wells. The rehydrating agarose does not significantly enter under the wells (at most a protruding lip around the rim of the well is observed occupying no more than $25 \%$ of the well-space), but rather flows around as they are machined to sit $50 \mu \mathrm{m}$ above the coverslip surface. The device is designed such that the hydrated agarose substrate layer is sufficiently thin for the evanescent field to penetrate through to the bilayer. In these experiments the rehydrating channels were filled with molten $2.5 \%$ agarose in TE buffer and allowed to set. The lipid in oil solution was pipetted into the wells. After 15 minutes an aqueous droplet of approximately $50 \mathrm{~nL}$ volume was pipetted into each well, whereupon the droplet sinks to the substrate agarose layer at the bottom of the well, and a bilayer spontaneously forms at the interface between the agarose substrate and the aqueous droplet, see Fig.1. We have not characterized the thickness or the viscosity of the aqueous layer between the supporting hydrogel and the bilayer, however the agarose layer must be thinner than $200 \mathrm{~nm}$ as we are able to excite fluorophores on the far side of the substrate gel with the TIRF evanescent field.

\subsection{Protein Preparation}

A single-cysteine mutant of $\alpha$-hemolysin with a C-terminal hexahistidine tag ( $\alpha \mathrm{HL}-\mathrm{T} 292 \mathrm{C}$ $\left.\mathrm{R}(\mathrm{GS})_{2} \mathrm{~S}(\mathrm{G})_{2}(\mathrm{~S})_{2}(\mathrm{H})_{6}\right)$ was prepared by overexpression in BL21(DE3)pLysS E. coli, using a pT7-SC1 vector ${ }^{2}$ 
by induction with $0.5 \mathrm{mM}$ IPTG. Following resuspension of the cell pellet in buffer (0.1\% (v:v) Triton X-100, $50 \mathrm{mM}$ Tris, $150 \mathrm{mM} \mathrm{NaCl}, 1 \mathrm{mM}$ PMSF, pH 8.0), cells were lysed by sonication on ice, and centrifuged to pellet the debris. The supernatent was purified using a Ni:NTA column (HiTrap Chelating HP). The hemolytic fraction was dialysed overnight at $4^{\circ} \mathrm{C}$ against buffer $(10 \mathrm{mM}$ sodium acetate, $1 \mathrm{mM}$ DTT and 1 mM EDTA, pH 5.2), resulting in a pure sample. DTT was removed by desalting through gel filtration with TE buffer (Superdex 200 10/300 GL column). Immediately after elution the sample was labeled by addition of 10-fold molar excess of Cy3B-maleimide for 15 minutes at room temperature. Following labelling, the reaction was quenched by dilution in buffer and incubated on ice (10 mM MES, pH 5.5). Free dye, labeledand unlabeled-monomeric protein were separated using a cation exchange column (HiTrap SPFF) and eluted using a $\mathrm{NaCl}$ gradient. All purification steps were performed by FPLC (Äkta Purifier). Proteins were stored in darkness at $4^{\circ} \mathrm{C}$ prior to experimentation.

For the preparation of heptameric $\alpha$-hemolysin $\left(\alpha \mathrm{HL}_{7}\right)$, the protein was prepared following the procedure decribed for monomer $\left(\alpha \mathrm{HL}_{1}\right)$, with the exception that $\mathrm{Cy} 3$-maleimide was used for labeling, and in contrast to the labeling reaction for the $\alpha \mathrm{HL}_{1}$ preparation, $\alpha \mathrm{HL}_{7}$ was not quenched by dilution in low $\mathrm{pH}$ buffer, but instead quenched by addition of excess 2-ME; as it was important to maintain a high concentration of protein for effective oligomerization. The concentrated sample was oligomerized using $6.25 \mathrm{mM}$ deoxycholate at $37^{\circ} \mathrm{C}$ for 16 hours, as previously described. ${ }^{3}$ The oligomerized sample was then subjected to electrophoresis (10\% Tris-HCl gel, $\mathrm{pH} 8.1$ with Tris-Tricine running buffer, $50 \mathrm{mM}$ Tris, $50 \mathrm{mM}$ Tricine, $\mathrm{pH}$ 8.3). A fluorescence gel imager (Gel Doc XR, Bio-Rad Laboratories, Hercules, CA) was used to identify the heptameric bands, which were subsequently excised, resuspended in TE buffer and filtered to remove the polyacrylamide using a microcentrifuge filter.

The separation of Cy3B-labeled and unlabeled $\alpha \mathrm{HL}_{1}$ protein by cation exchange chromatography is shown in Supp Fig.1, A. From the relative absorbance of the first eluting peak, at 280 and $560 \mathrm{~nm}$, factoring out the absorbance contribution of the dye at $280 \mathrm{~nm}$, we estimate an approximate labeling stoichiometry of 1:1. The labeled and unlabeled monomers were not purified before oligomerization, as it is not critical to have all monomer subunits of the heptamer labeled for tracking. The $\alpha \mathrm{HL}_{7}$ protein was purified directly from the gel, shown in Supp Fig.1, B. 

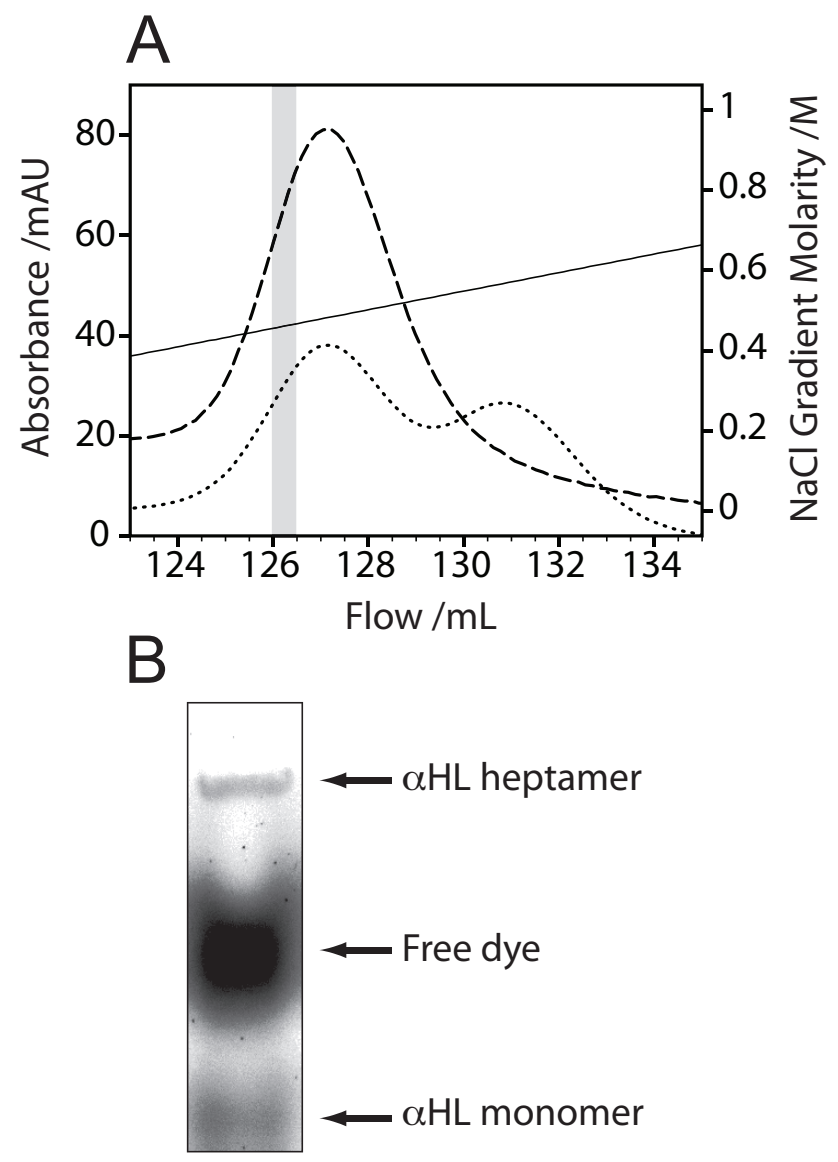

Figure 1: (A) Cation exchange chromatogram showing the elution profile of labeled and unlabeled monomeric $\alpha$-hemolysin. Absorbance at $280 \mathrm{~nm}$ (dotted line), absorbance at $560 \mathrm{~nm}$ (dashed line) and the concentration of the eluent $\mathrm{NaCl}$ (solid line) are shown on the $y$ axes. The $0.5 \mathrm{~mL}$ fraction used in subsequent experiments is shown between as a gray band. (B) Fluorescence image of the SDS-PAGE gel used to separate labeled heptameric $\alpha$-hemolysin $\left(\alpha \mathrm{HL}_{7}\right)$, free dye and non-oligomerized monomeric $\alpha$-hemolysin $\left(\alpha \mathrm{HL}_{1}\right)$. 


\section{Tracking experiments}

A $15 \mathrm{~mW} 532 \mathrm{~nm}$ circularly polarised, cw laser (Compass 215M, Coherent Inc, Santa Clara, CA) was focussed at the back aperture of an oil immersion objective lens (60x Plan Apo N.A. 1.4, Nikon Instruments, UK) mounted on an inverted microscope (TE-2000, Nikon Instruments, UK), illuminating the analytes through TIR at the coverslip surface. The emitted fluorescence was collected through the same objective, transmitted through dichroic (Q565LP) and band-pass (HQ580/60m) filters (Chroma Technology Corp, Rockingham, VT), and imaged using a $128 \times 128$ pixel frame-transfer emCCD detector (iXon DU-860, Andor Technology PLC, Belfast, UK). Images were collected at $2.5 \mathrm{~ms}$ intervals and calibrated to $212 \mathrm{~nm}$ in each dimension per pixel. Image sequences were converted to 1000-frame stacked 16-bit Tagged Image File Format (TIFF) bitmaps.

Protein lateral diffusion experiments were performed in DHBs created in exactly the same manner as for our fluorophore tagged lipid diffusion experiments, incorporating $10 \mathrm{pM}$ concentration labeled proteins in TE buffer. All experiments were carried out at room temperature.

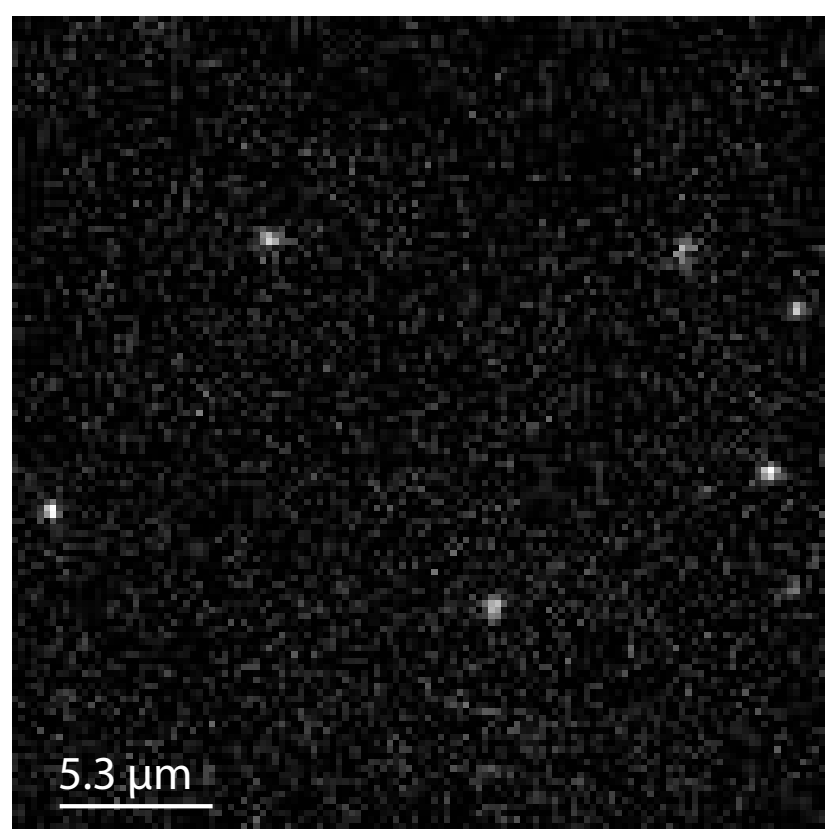

Figure 2: A representative image of fluorescently labelled $\alpha \mathrm{HL}_{7}$ diffusing on a DHB. This raw $128 \times 128$ pixel image was acquired from a $2.5 \mathrm{~ms}$ exposure time and is shown without any filtering or adjustment. 


\subsection{Fluorophore Tracking Algorithm}

We implement a new algorithm for the extraction of single-particle tracks from single-molecule fluorescence images. Our method relies upon the astronomical algorithm CLEAN. ${ }^{4,5}$ In radioastronomy, objects formed in astronomical images are represented as scaled point-spread functions. CLEAN is used to detect point-spread functions by iterative removal of the brightest spot in an image where there is relatively poor Fourier plane coverage. ${ }^{6}$ As the fluorescence from a single-molecule is also imaged as a diffraction limited spot, the CLEAN algorithm can also be applied. Due to its simplicity, CLEAN spot detection provides a straightforward and computationally inexpensive alternative method for fluorescence SPT.

\subsubsection{Initial Image Analysis}

An optimal threshold for discriminating between fluorescence spots and noise is determined by generating a histogram of all pixel intensities in the entire sequence of images (the image-stack). A user-estimated number of spots and their average diameter is taken to establish a threshold percentage of pixels to be counted as fluorescence used by CLEAN for analysis. This provides a simple and accessible method to assign a correct noise threshold. In these experiments, this corresponded to a minimum signal-to-noise ratio of 4:1.

\subsubsection{Spot Detection}

The iterative CLEAN algorithm is implemented as described by Clark: ${ }^{5}$ CLEAN first finds the brightest pixel in the image. A $12 \times 12$ pixel region of interest centered on this pixel (the patch-area) is then extracted from the image. Given that the point spread function of a fluorophore in our images covers approximately $3 \times 3$ pixels, this $12 \times 12$ pixel patch-area facilitates accurate fitting. A 2 D Gaussian function is then fitted to the spot, using an optimized Levenberg-Marquardt algorithm ${ }^{7}$ and the fitting parameters are recorded. Gaussian fitting has been shown to provide the most accurate method for localizing singlemolecule fluorescence spots. ${ }^{8,9}$ From this procedure we determined the position of the fluorophore in the images with mean accuracy of $10.6 \mathrm{~nm}$. A Gaussian mask is constructed from the fit parameters and the resulting function is subtracted from the original image, removing the spot. If the minimization fails, the patch-area is replaced with background intensity, hence disregarding that spot. CLEAN then iterates to find the next brightest pixel, which is again fitted with a 2D Gaussian until the noise threshold is reached. We recycle the Gaussian parameters recovered from the first fit to estimate the initial fitting parameters for the 
next spot in order to improve algorithm efficiency. Finally, an array is constructed with time-stamped spot localizations and fitting errors for all parameters.

\subsubsection{Track Reconstruction}

Vector distances between all spots in the $\mathrm{n}^{\text {th }}$ and $(\mathrm{n}+1)^{t h}$ frames are calculated. Tracks are then created by linking each spot with its closest partner in the subsequent frame by minimizing the total inter-spot distance. Tracks are terminated for inter-spot distances greater than the user-defined maximum allowed displacement (954 nm) between subsequent images. This process occurs iteratively throughout the whole image-stack, linking the spot-positions together to form tracks. Tracks shorter than the user-defined minimum track length (8 frames) are discarded. The fluorophore tracking algorithm was implemented in Java (version 1.5) integrated as a plugin into the open-source image analysis tool ImageJ (version 1.37). ${ }^{10}$

The implementation of the CLEAN algorithm we present is efficient at detecting and localizing fluorescence spots in images. In particular, this algorithm is robust enough that we can track many hundreds of spots simultaneously through a stack of many thousands of images. This is in contrast to other tracking techniques such as those based on Bayesian particle filters, ${ }^{11-13}$ which although able to work with lower signal-to-noise ratios and missing spots, have a greater computational burden and can analyze only limited numbers of concurrent spots. Using our algorithm, a typical analysis of a 1000 frame image of diffusing particles takes less than 3 minutes on a single desktop computer. 


\section{Data Analysis}

\subsection{Lateral Diffusion Coefficient Calculations}

Mean square displacements (MSDs) were calculated for each individual track using the running-window method as described by Qian et al. ${ }^{14}$ and Saxton ${ }^{15}$ where the MSD for a single spot of a particular time-lag $\left(\Delta t_{n}\right)$ is,

$$
\operatorname{MSD}\left(\Delta t_{n}\right)=\frac{1}{N-n+1} \sum_{j=0}^{N-n}\left([x(j \delta t+n \delta t)-x(j \delta t)]^{2}+[y(j \delta t+n \delta t)-y(j \delta t)]^{2}\right)
$$

This calculation results in the average over all pairs' squared two-dimensional displacement, for all overlapping frames $N-n+1$, where $N$ is the total number of frames in the track, and $n$ is the window size, such that $n \delta t=\Delta t_{n}$, and where $\delta t$ is the time step between frames. Lateral diffusion coefficients $D_{\text {lat }}$ are calculated for each individual track by fitting the MSD vs. $\Delta t_{n}$ plot with a linear function weighted by the standard deviation, assuming our errors are approximately normally distributed. The gradient of the linear fit is $4 D \Delta t_{n}$. The histogram of the $\mathrm{D}_{\text {lat }}$ values for the individual tracks can be fitted with the probability density function of the gamma distribution, weighted by the square-root of the data,

$$
P\left(D_{\text {lat }}\right)=\frac{A D_{\text {lat }}^{\kappa-1}}{\Gamma(\kappa) \theta^{\kappa}} \exp \left[\frac{-D_{\text {lat }}}{\theta}\right]
$$

where $\Gamma$ is the gamma function and $A, \theta$ and $\kappa$ are the normalisation, scale factor and shape factors respectively.

\subsection{Population Multi-Component Analysis}

In our study we analyzed all trajectories without any selection criteria, other than minimum track length and maximum inter-observation displacement distance, in order to accurately characterize all mobility components and verify our new tracking procedure without bias.

It has been shown that where multiple mobility components are present in a population of trajectories, a simple MSD calculation is insufficient to describe the population's diffusive behavior. ${ }^{16-18}$ In order to accurately gauge the lateral mobilities and fraction sizes of different mobility components in the population, 
it is useful to calculate the probability that a particle is to be found within a circle of radius $r$ at time $t$. This is given by the multiexponential equation

$$
P\left(r^{2}, t\right)=1-\sum_{i=1}^{C} \alpha_{i} \exp \left[-\frac{r^{2}}{r_{i}^{2}(t)}\right]
$$

where $\alpha_{i}$ is a fraction of tracks belonging to a single mobility component, $i, \mathrm{C}$ is the number of mobility components and

$$
\sum_{i=1}^{C} \alpha_{i}=1
$$

The lateral diffusion coefficient of a mobility component can then be calculated from

$$
r_{i}^{2}(t)=4 D_{l a t, i} t
$$

To calculate $P(r, t)$ from our experimental results, we counted the number of square displacements with values less than or equal to $r^{2}$ normalized by the total number of data points for each time-lag $t$, where the minimum $r^{2}$ step size was set to $1 \mathrm{~nm}$. Time-lags were set at $20 \mathrm{~ms}$ (where all tracks are included), $40 \mathrm{~ms}$, $80 \mathrm{~ms}$ and $160 \mathrm{~ms}$. By plotting $1-P$ versus $r^{2}$ and fitting to Equation 3 the mobility component fractions are obtained.

The trajectories were best fit with biexponential functions, rather than mono- or triexponential functions, judged by comparison of reduced chi-squared $\left(\chi_{r}^{2}\right)$. The biexponential analysis was adequate for all timedelays.

No slow component $\left(D_{\text {lat }} \lesssim 1.5 \mu \mathrm{m}^{2} \mathrm{~s}^{-1}\right)$, corresponding to being hindered, was seen in our $\alpha \mathrm{HL}_{7}$ analysis. This could be due to an insufficient number of data points necessary for an accurate triexponential fit.

All statistical analyses were performed in IGOR Pro (Wavemetrics, Portland, OR). 

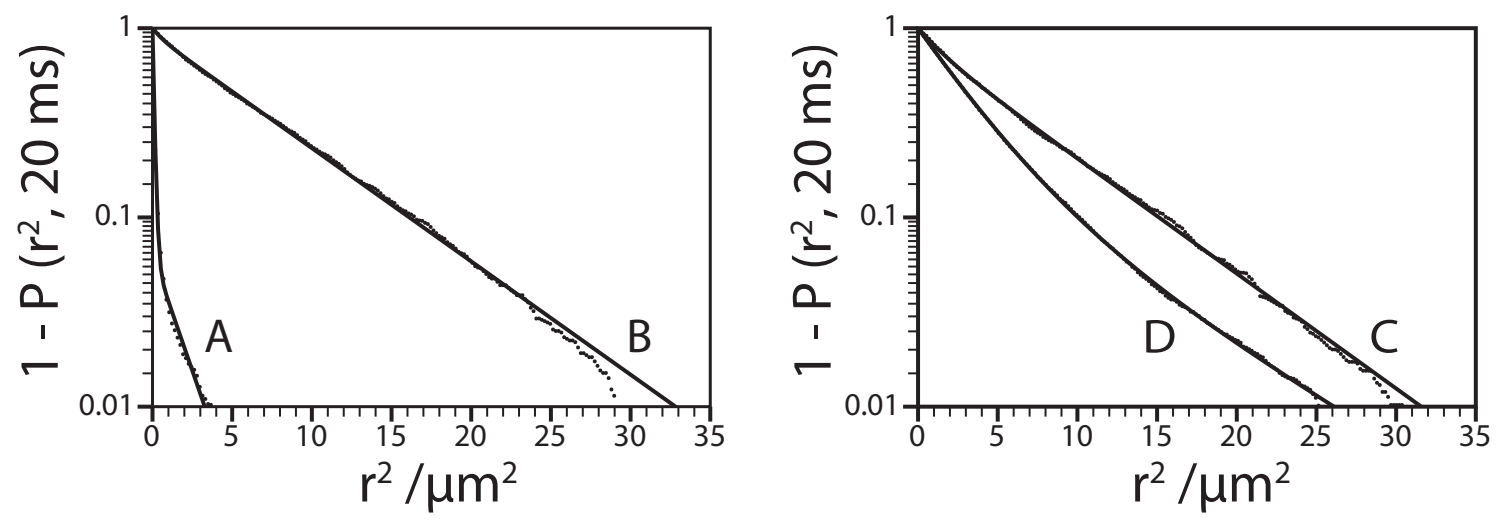

Figure 3: Graphs showing 1 - $\mathrm{P}$ vs. $r^{2}$, where $\mathrm{P}$ is the probability that a particle will be found at a position defined by the radius $r^{2}$ after $20 \mathrm{~ms}$ of diffusion. Only 200 data points are shown for clarity, see Table 1 for information on data set sizes. (A) SLB lipids, (B) DHB lipids, (C) $\alpha \mathrm{HL}_{1}$ proteins and (D) $\alpha \mathrm{HL}_{7}$ proteins. 


\section{References}

1. Cremer, P. S.; Boxer, S. G. J. Phys. Chem. B 1999, 103, 2554-2559.

2. Cheley, S.; Malghani, M. S.; Song, L.; Hobaugh, M.; Gouaux, J. E.; Yang, J.; Bayley, H. Protein Eng. 1997, 10, 1433-1443.

3. Song, L.; Hobaugh, M. R.; Shustak, C.; Cheley, S.; Bayley, H.; Gouaux, J. E. Science 1996, 274, $1859-1866$.

4. Högbom, J. A. Astronomy and Astrophysics Supp 1974, 15, 417.

5. Clark, B. Astronomy and Astrophysics 1980, 89, 377-378.

6. Thompson, A. R.; Moran, J. M.; Swenson, G. W. Interferometry and synthesis in radio astronomy; Wiley-Interscience, New York, 2001.

7. Lampton, M. Computers in Physics 1997, 11, 110-115.

8. Thompson, R. E.; Larson, D. R.; Webb, W. W. Biophys. J. 2002, 82, 2775-2783.

9. Cheezum, M. K.; Walker, W. F.; Guilford, W. H. Biophys. J. 2001, 81, 2378-2388.

10. Abramoff, M. D.; Magelhaes, P. J.; Ram, S. J. Biophotonics International 2004, 11, 36-42.

11. Smal, I.; Niessen, W.; Meijering, E. Nonlinear Statistical Signal Processing Workshop 2006, 44.1-44.4.

12. Smal, I.; Niessen, W.; Meijering, E. IEEE Conference Proceeding: 3rd IEEE International Symposium on Biomedical Imaging: Macro to Nano 2006, 550-553.

13. Smal, I.; Niessen, W.; Meijering, E. IEEE Conference Proceeding: 4th IEEE International Symposium on Biomedical Imaging: Nano to Macro 2007, 1048-1051.

14. Qian, H.; Sheetz, M. P.; Elson, E. L. Biophys. J. 1991, 60, 910-921.

15. Saxton, M. J. Biophys. J. 1997, 72, 1744-1753.

16. Schütz, G. J.; Schindler, H.; Schmidt, T. Biophys. J. 1997, 73, 1073-1080.

17. Sonnleitner, A.; Schutz, G.; Schmidt, T. Biophys. J. 1999, 77, 2638-2642. 
18. Lommerse, P. H. M.; Vastenhoud, K.; Pirinen, N. J.; Magee, A. I.; Spaink, H. P.; Schmidt, T. Biophys. J. 2006, 91, 1090-1097. 